\title{
Interpretation of the anisotropy of $M$ MO radiation in slow I on Au collisions $\dagger$
}

\author{
H Hartung $\ddagger$, B Fricke $\ddagger$, T Morović $\ddagger$, W-D Sepp $\ddagger$ and A Rosén $\S$ \\ ‡esamthochschule Kassel, Theoretische Physik, D-3500 Kassel, Germany \\ $\S$ Physics Department, Chalmers University of Technology, Göteborg, Sweden
}

Received 20 October 1978, in final form 29 December 1978

\begin{abstract}
A comparison between experimental and calculated spectral shape and energy dependence of the M MO x-ray anisotropy in heavy-ion collisions of $\mathrm{I}$ on Au is presented. The calculation is performed within the kinematic-dipole model of anisotropy using MO $\mathrm{X}$-rays determined from SCF relativistic correlation diagrams.
\end{abstract}

\section{Introduction}

Non-characteristic $\mathrm{x}$-ray anisotropies in heavy-ion collisions have recently been measured by many groups (Kraft et al 1974, Greenberg et al 1974, Meyerhof et al 1975, Wölfli et al 1975, 1976, 1977, Folkmann et al 1976, Frank et al 1976a, b). Several different theoretical approaches have been used in efforts to understand the details of the complicated behaviour of the experimental anisotropies (Müller and Greiner 1974, Briggs and Dettmann 1977, Gros et al 1977, Hartung and Fricke 1978, Anholt 1978). It is shown here that the kinematic-dipole model (Hartung and Fricke 1978) of the anisotropy together with a realistic SCF many-electron correlation diagram (Fricke et al 1976) for the I-Au system is able to reproduce the measured M MO structure of the anisotropy. This is a considerable success since the detailed behaviour of many electronic $\mathrm{M}$ and $\mathrm{N}$ levels with several important pseudocrossings must be considered in the interpretation. For the $\mathrm{K}$ MO region, where the number of levels and pseudocrossings is very small, a coupled-channel calculation for the time-dependent occupation amplitudes has been used successfully (Fritsch and Wille 1977, Briggs and Taulbjerg 1976). In the M MO region not only the large number of orbitals but also the accuracy of the wavefunctions involved do not allow similar coupled-channel calculations.

The second main difference from the $\mathrm{K}$ Mo region is that the influence of rotational coupling on the anisotropy is unimportant. The distances of closest approach discussed here remain too large for effective rotational coupling among the $3 p$ and $3 d$ levels. The hole transfer into the inner levels is dominated by several radial couplings. Kraft et al (1974) and Folkmann et al (1976) have made experimental investigations of the I-Au system analysed in this paper.

† Work partially supported by Gesellschaft für Schwerionenforschung, Darmstadt, Germany. 


\section{Method}

At impact energies of a few MeV for I on Au used in the experiments (Kraft et al 1974, Folkmann et al 1976) the velocities of the nuclei $v_{\mathrm{N}}$ are much smaller than the velocities $v_{\mathrm{e}}$ of the inner electrons, so the adiabaticity parameter $v_{\mathrm{N}} / v_{\mathrm{e}}$ is very small. For $17 \mathrm{MeV}$ I on Au for example, the adiabaticity parameter values are $0.02,0.04$ and 0.07 for the $\mathrm{K}, \mathrm{L}$ and $\mathrm{M}$ electrons, respectively. This shows that the system as a whole is highly adiabatic with the consequence that the general picture given by a correlation diagram is a very good starting point for the analysis of the anisotropy of the non-characteristic radiation observed in the experiments.

The intensity function $S(\boldsymbol{k}, \boldsymbol{R})$ which gives the number of spontaneously emitted photons from a quasimolecular system is a function of the photon propagation vector $k$ and the relative distance vector $\boldsymbol{R}$ of the two nuclei (Fricke et al 1976):

$$
S(\boldsymbol{k}, \boldsymbol{R})=\sum_{i<j} g_{i} g_{j} f_{i}\left(1-f_{j}\right) P_{i j} Y_{i j}(\boldsymbol{k} \cdot \boldsymbol{R}) D_{i j}
$$

where $\boldsymbol{R}$ in the adiabatic case is used as a parameter; $g_{i}$ and $g_{j}$ are molecular level degeneracies; and $f_{i}$ and $f_{j}$ are occupation numbers of the electronic states. $P_{i j}$ is the transition probability between the states $|i\rangle$ and $|j\rangle$ with $E_{i j}$ the transition energy and $Y_{i j}(\boldsymbol{k} . \boldsymbol{R})$ the angular characteristic of the transition involved. To a good approximation $D_{i j}$ is given as a Lorentz distribution centred around the transition energy $E_{i j}$. The halfwidth of the Lorentzian refers to the collision broadening of the transition and represents the dynamic part of the collision process in which the radiation occurs. In a general treatment $D_{i j}$ turns out to be equivalent to the Fourier components representing the time dependence of the transition energies (Weisskopf 1932). In other words, $D_{i j}$ is the dynamical correction to the approximation that $R$ is taken to be a parameter instead of a dynamical variable.

In the experiment the total intensity emitted during the collision process is measured as a function of the laboratory angle $\theta$ or photon propagation vector $\boldsymbol{k}$. Equation (1) should therefore be summed over all internuclear distances $\boldsymbol{R}$ to get the corresponding total intensity

$$
S(\boldsymbol{k})=\iiint \mathrm{d}^{3} R S(\boldsymbol{k}, \boldsymbol{R}) W(\boldsymbol{R})=S\left(\theta, E_{\mathbf{x}}\right)
$$

where $E_{\mathbf{x}}=c \hbar|\boldsymbol{k}|$ is the photon energy. $W(\boldsymbol{R})$ is the weight function, which represents the probability of finding the system in the relative position $\boldsymbol{R}$. It is not only determined from geometric considerations but also depends on the internuclear potential via the kinetic energy at every point.

The incoherent summation in equation (2) is expected to be a good approximation for the 28 transitions used here. In addition, every transition often contributes at several radial distances $R$, where the transition energies coincide. Due to the large number of interferences one can expect with certainty that all interference effects can be neglected in contrast to the $\mathrm{K}$ MO few-level system discussed in Briggs and Dettmann (1977). Using this function $S\left(\theta, E_{\mathrm{x}}\right)$ it is possible to calculate the anisotropy which usually is defined by

$$
A\left(\theta_{1}, \theta_{2}, E_{\mathrm{x}}\right)=\left(S\left(\theta_{1}, E_{\mathrm{x}}\right) / S\left(\theta_{2}, E_{\mathrm{x}}\right)\right)-1 .
$$




\section{Numerical treatment and physical approximations}

The three integrations of equation (2) which are most consistent with the physical problem are (see figure 1): (i) along the trajectory $R(\psi)$; (ii) over all impact parameters $b$; and (iii) over all scattering-plane angles $\phi$ relative to the detector at $90^{\circ}$ which fixes the plane of observation. The last integration can be done analytically since only trigonometric functions are involved via the angular distribution functions $Y_{i j}(\boldsymbol{k} . \boldsymbol{R})$. The first two integrations are calculated numerically with a simple three-point Simpson integration method and 97 points in total along one Rutherford trajectory which is fast and accurate enough for the $\mathrm{M}$ MO transition energies. Both integrations over the trajectories and the impact parameters can be cut off at relatively small distances where the transition energies are already smaller than the $M$ MO transition energies of interest. In our case this is the region between 6 and $8.5 \mathrm{keV}$.

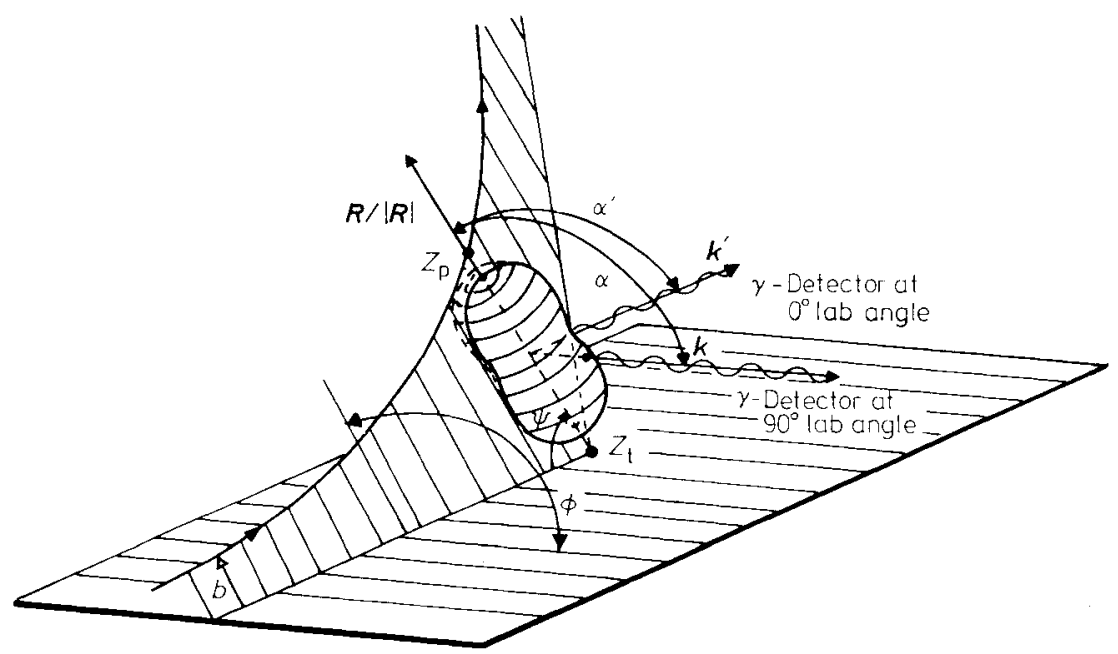

Figure 1. Schematic view of the collision of the projectile nucleus $Z_{\mathrm{p}}$ and the target nucleus $Z_{\mathrm{t}}$. The angular characteristic for a dipole transition proportional to $1+\cos ^{2} \alpha$ is shown.

In the actual calculations the following approximations have been made.

(i) We restrict ourselves to dipole transitions between the levels. This implies that the angular distribution function (Jackson 1975) $Y_{i j}(\boldsymbol{k}, \boldsymbol{R})$ from equation (1) is either $\sin ^{2} \alpha$ or $\left(1+\cos ^{2} \alpha\right)$, where $\alpha$ is the angle between the direction of the photon $k$ and the internuclear distance $\boldsymbol{R}$ (see figure 1). The first of the two functions has to be used for transitions with $\Delta \Omega=0$, the second for transitions with $\Delta \Omega= \pm 1 \dagger$.

(ii) The wavefunctions are sufficient to give accurate binding energies but not good enough to give accurate transition-dipole probabilities. Therefore $P_{i j}$ was taken to be proportional to the cube of the transition energy between the states $|i\rangle$ and $|j\rangle$, which is the leading term of the dipole matrix element for the $\mathrm{x}$-ray energies under discussion. The remaining matrix element is set to unity for all transitions because only relative values of the transition probabilities are relevant.

\footnotetext{
+ Concerning the notation of the MO levels we write the $\Omega$ quantum number of the component of the angular momentum along the symmetry axis as index of the level member. We therefore write e.g. $7_{(1 / 2)}$ for the 7 th level with $\Omega=\frac{1}{2}$ (see also figure 2 ).
} 
(iii) The collision broadening was taken into account by establishing a constantenergy window width of $0.5 \mathrm{keV}$ for the photon energies $E_{\mathrm{x}}$. A much more realistic procedure is the lineshape description as given by Anholt (1976). Actual comparison of both methods shows only very small differences in the result. So our method was chosen as a good approximation to simplify the integration. This approximation becomes questionable near the united-atom limit where transitions into only one level contribute at a given $\mathrm{x}$-ray energy ( $\mathrm{K}$ MO case). In contrast to this we have many contributions from various levels at many internuclear distances. Therefore the united-atom limit does not play the same important role as in the $\mathrm{K}$ MO case.

(iv) The transition energies were extracted from an adiabatic relativistic SCF correlation diagram given by Fricke et al (1976). The relevant part is shown in figure 2 .

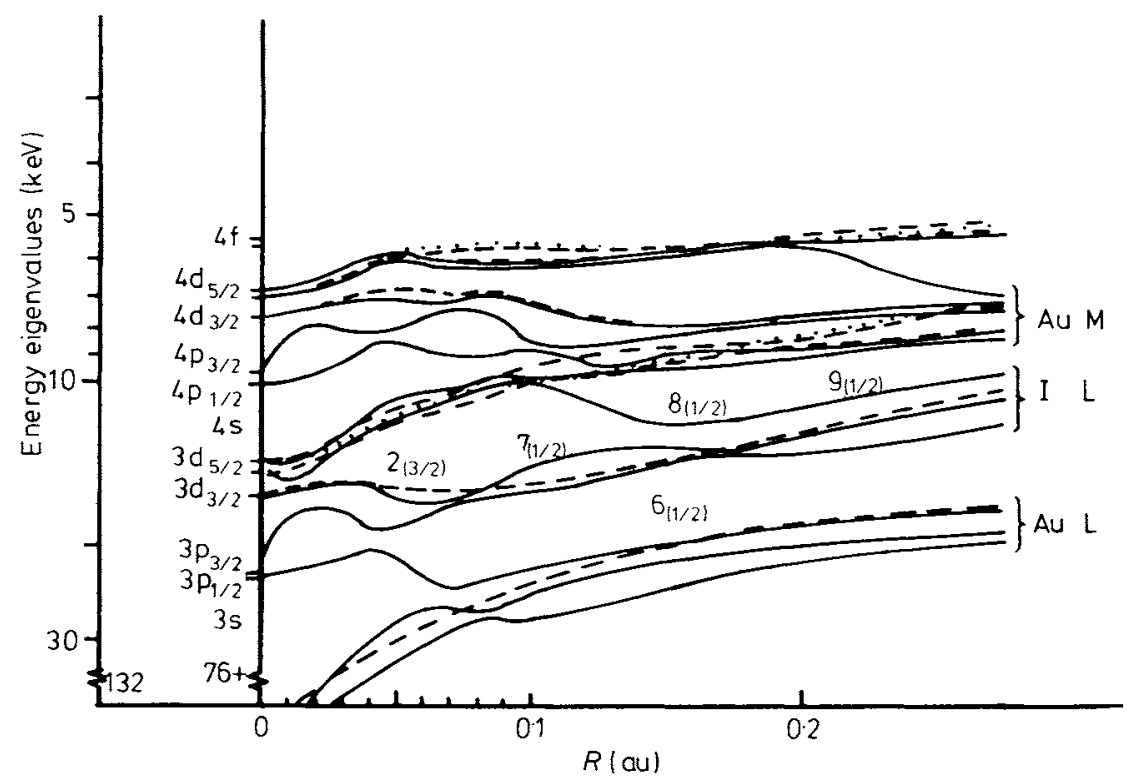

Figure 2. Part of the correlation diagram for the system $I \rightarrow A u$. The levels of interest are numbered within the same component of the total angular momentum $\Omega$ which also is included in parentheses.,$- \Omega=\frac{1}{2} ;---, \Omega=\frac{3}{2} ; \ldots, \Omega=\frac{5}{2}$.

(v) The relative nuclear motion is described by Coulomb trajectories in the field of the effective nuclear charges $Z_{1}^{*}$ and $Z_{2}^{*}$. An exact trajectory calculated from the internuclear potential of the SCF calculation is in principle possible (Morovic et al 1978) but is unrealistic in this work due to the long computer time involved.

(vi) The vacancy distribution in the $\mathrm{M}$ and $\mathrm{N}$ shells of the quasi-atom is very important in the interpretation of $\mathrm{M} M O \mathrm{x}$-rays. Since there are no ab initio calculations for the hole distribution of such a complicated system these quantities can only be approximated with the help of Landau-Zener calculations (Landau 1932, Zener 1932). We have performed such calculations for the radial couplings between the $9_{(1 / 2)}$ and $8_{(1 / 2)}$ levels at $0 \cdot 1$ au and for the most important coupling of the $8_{(1 / 2)}$ level with the $7_{(1 / 2)}$ and $6_{(1 / 2)}$ levels at about $0.02 \mathrm{au}$. The results for this last crossing may include some errors, because we are dealing with three crossings not well separated. We calculate the transition probability for every energy and impact parameter $b$. After the integration 
over $b$ we get an averaged probability of less than $1 \%$ for the transfer of the holes in the $6_{(1 / 2)}$ plus $7_{(1 / 2)}$ levels from the $8_{(1 / 2)}$ level. For the hole distribution among the $6_{(1 / 2)}$ and $7_{(1 / 2)}$ levels we use a $1: 1$ ratio which finally leads to a $1: 1: 250$ relation for the vacancy distribution within the $6_{(1 / 2)}, 7_{(1 / 2)}$ and $8_{(1 / 2)}$ levels, respectively.

In addition to the calculations discussed in (vi) the following remarks may further clarify the situation. Both the $\mathrm{M}$ - and $\mathrm{N}$-shell holes will be fed from outer-shell vacancies into the inner $\Omega=\frac{1}{2}$ levels by radial coupling. Even if we assume an initial homogeneous hole distribution in the $\Omega=\frac{1}{2}$ and $\Omega=\frac{3}{2}$ levels a large number of holes will be created in the $\Omega=\frac{1}{2}$ levels by an electron promotion mechanism along the incoming part of the trajectory. The $\Omega=\frac{3}{2}$ levels behave much more smoothly and have almost no crossings with each other so that no electron transfer to very high levels may occur via radial coupling. Taking this into consideration, we can therefore assume, as general behaviour, a relatively higher electron population of the $\Omega=\frac{3}{2}$ levels compared with the $\Omega=\frac{1}{2}$ level population at small internuclear distances, which shows that the transitions from populated $\Omega=\frac{3}{2}$ levels into the inner $\Omega=\frac{1}{2}$ levels are predominant.

Along the incoming part of the trajectory the number of holes in the levels originating from the I L shell is much smaller than in the levels from the $\mathrm{Au} \mathrm{M}$ shell. This can be explained, firstly because the Coulomb ionisation cross section is strongly dependent on the binding energy and secondly because various levels from the Au M shell are diabatically connected with higher shells of the united system. Because of the very narrow crossing between the $8_{(1 / 2)}$ and $9_{(1 / 2)}$ levels at $0 \cdot 1$ au nearly all holes will be transferred from the $9_{(1 / 2)}$ into the $8_{(1 / 2)}$ level in the region $0 \cdot 02 \leqslant R \leqslant 0 \cdot 1$ au. For impact parameters, where the radial coupling at $R \simeq 0.02$ au is not reached, the holes will not remain in the $8_{(1 / 2)}$ level, but they will be transferred back to the $9_{(1 / 2)}$ level via the same crossing at about $0 \cdot 1$ au. Experimentally the $\mathrm{x}$-ray cross section of $\mathrm{Au} \mathrm{M}$ radiation is also about two orders of magnitude larger than $I L$ radiation (Hagmann 1977). This indicates a higher hole population at the beginning of the process in the levels originating from the Au M shell, if we also assume that asymptotically only a few holes will remain in the $8_{(1 / 2)}$ level. For impact parameters, where a radial coupling to the $7_{(1 / 2)}$ and $6_{(1 / 2)}$ levels from the $8_{(1 / 2)}$ levels is possible we get an averaged hole distribution of $1: 1: 250$ (see (vi)). So the $7_{(1 / 2)}$ and $6_{(1 / 2)}$ levels will contribute to the spectrum only for these small impact parameters. There will be no, or very little, contribution from transitions into the $2_{(3 / 2)}$ level, because they will only be fed with holes by rotational coupling for even smaller impact parameters. This means that the number of holes for the $2_{(3 / 2)}$ level averaged over all impact parameters which contribute to the spectrum will be even smaller than those of the $6_{(1 / 2)}$ and $7_{(1 / 2)}$ levels.

These arguments definitely show that most of the transitions will occur from the high $\Omega=\frac{3}{2}$ levels into the 6 th, 7 th and 8 th $\Omega=\frac{1}{2}$ levels. These transitions have $\Delta \Omega= \pm 1$. Of course, the transitions between the higher and lower $\Omega=\frac{1}{2}$ levels cannot be totally neglected, but their contributions are isotropic in the extreme relativistic limit, which we deal with here. Thus, they do not directly contribute to the anisotropy.

\section{Results and discussion}

The resulting spectrum of such a calculation for $17.7 \mathrm{MeV}$ I on $\mathrm{Au}$ is shown in figure 3 together with the experimental spectrum of Kraft et al (1974). Only the fall-off behaviour on the high-energy side does not compare very well with the experiment. The reason for this behaviour is easy to understand. For computational reasons we have 
used a constant hole distribution in the levels $6_{(1 / 2)}$ and $7_{(1 / 2)}$ for all impact parameters. But in reality the number of holes in these levels will be negligible for impact parameters $b \geqslant 0.02$ au. This unrealistic assumption reproduces exactly the main part of the spectrum above $8 \mathrm{keV}$ in the theoretical spectrum. The spectral anisotropies for iodine impact energies $E_{0}=6 \cdot 3-25 \cdot 1 \mathrm{MeV}$ determined from the calculated spectra are shown in figure 4 together with the experimental values (Folkmann et al 1976). In the experiment measurements were made at $15^{\circ}$ and $90^{\circ}$. We therefore compare the calculated anisotropies at the same angles. The following properties can be seen.

(i) The computed anisotropies show a peak structure around $E_{\mathrm{x}}=7 \mathrm{keV}$ except for the diagram with $E_{0}=6.3 \mathrm{MeV}$, where a distinct minimum occurs.

(ii) The anisotropy peaks have maximal values between $E_{0}=11.2 \mathrm{MeV}$ and $17 \cdot 6 \mathrm{MeV}$ similar to the behaviour in the experiment. They also decrease slowly with increasing impact energy.

(iii) For impact energies above $11 \mathrm{MeV}$ the positions of the anisotropy peaks are located consistently at about $7 \mathrm{keV}$, which within the range of experimental errors is in

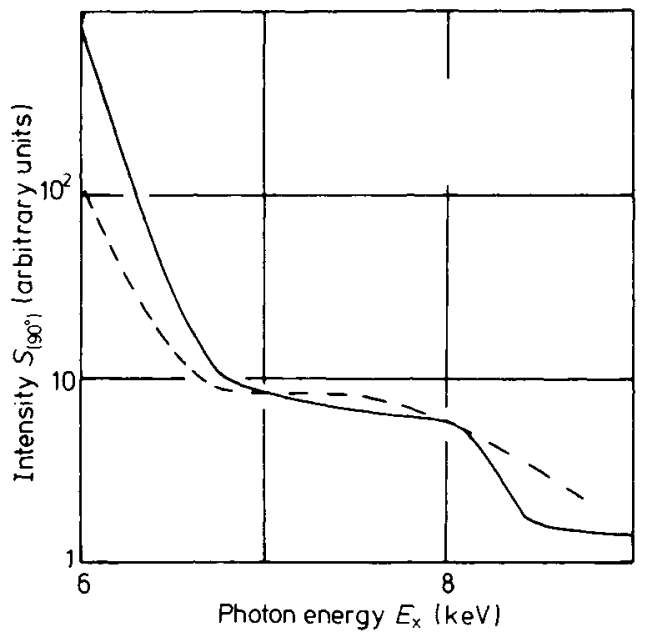

Figure 3. Calculated $\mathrm{M}$ MO spectrum for $17 \cdot 6 \mathrm{MeV}$ $\mathrm{I} \rightarrow \mathrm{Au}$. Only transitions into the $6_{(1 / 2)}, 7_{(1 / 2)}$ and $8_{(1 / 2)}$ levels are included. The experimental points are taken from Folkmann et al (1976). theoretical, ---, experimental.

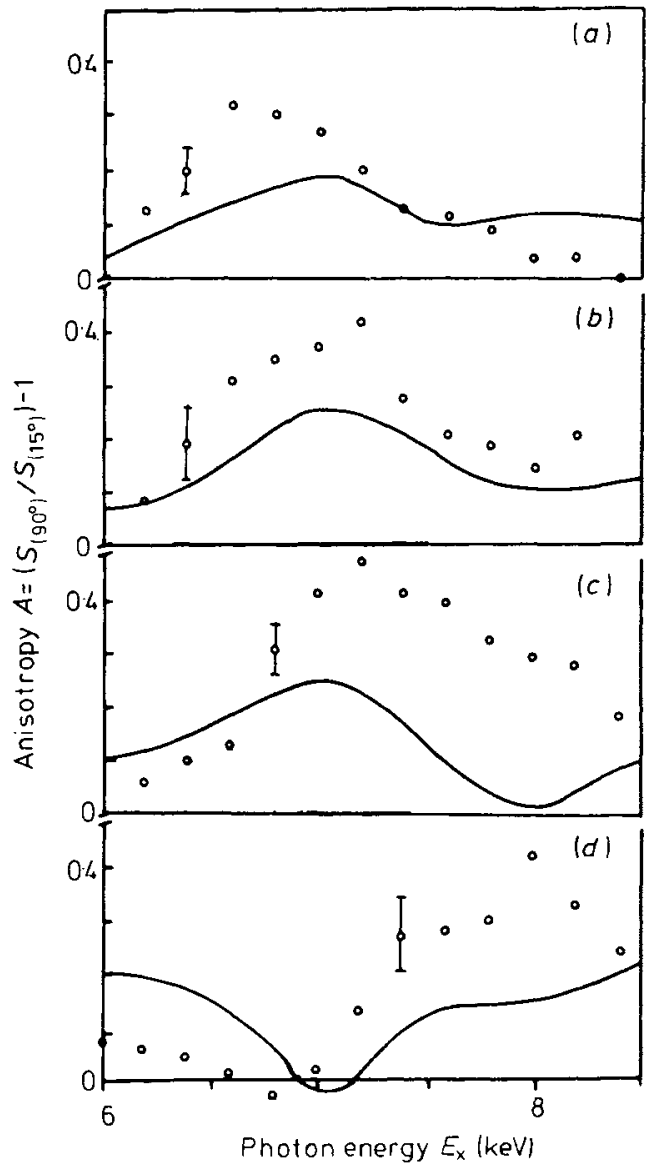

Figure 4. Spectral M MO anisotropies for different impact energies $E_{0}:(a) E_{0}=25 \cdot 1 \mathrm{MeV}$; $(b) E_{0}=$ $17.6 \mathrm{MeV}$; (c) $E_{0}=11.2 \mathrm{MeV}$; (d) $E_{0}=6.3 \mathrm{MeV}$. The experimental points are taken from Folkmann et al (1976). 
accordance with the experiment (see figures 2 and 4 of Folkmann et al 1976). For $E_{0}=6 \cdot 3 \mathrm{MeV}$ they interpret the structure above $7 \mathrm{keV}$ as a peak. These data may also be interpreted as a large plateau as found in the calculated anisotropy spectrum.

Because the anisotropy spectrum is a complicated superposition of many transitions, each with a different energy dependence, it is nearly impossible to provide a physical understanding of this structure. Nevertheless, we attempt to give an interpretation of the main features.

The large change in the anisotropy spectrum between $E_{0}=6.3$ and $11.4 \mathrm{MeV}$ results from decreasing transition energies into the $8_{(1 / 2)}$ level at internuclear distances above $0.015 \mathrm{au}$. For this small impact energy the distance of closest approach (about 0.02 au at $E_{0}=11 \mathrm{Mev}$ and 0.03 au at $E_{0}=6 \mathrm{MeV}$ ) is too far from the $8_{(1 / 2)}$ minimum at about 0.01 au. Only head-on collision trajectories which lead to negative anisotropy contributions reach the transition energy range under consideration. The anisotropy is therefore shifted to much smaller photon energies and the maximum decreases.

At about $11 \mathrm{MeV}$ the $8_{(1 / 2)}$ minimum is nearly reached in head-on collisions. The negative contributions to the anisotropy give the fall-off on the high-energy side of the spectrum above $7 \mathrm{keV}$. The large anisotropy contribution around $7 \mathrm{keV}$ arises from transitions into the $8_{(1 / 2)}$ minimum for non-zero impact parameters. This leads to higher intensities and a large anisotropy. Up to now we do not have any explanation for the relatively large discrepancy between experiment and our theory at about $8 \mathrm{keV}$ although the trend is reproduced. Observe that the experimental error bars are also quite large. For even larger impact energies the anisotropy slowly decreases because for even larger impact parameters the $8_{(1 / 2)}$ minimum is passed. A wide range of constant transition energies is obtained which lead to an isotropic radiation with high intensity along the single trajectory. These isotropic contributions become relevant for $E_{0}>20 \mathrm{MeV}$ and decrease the anisotropy with increasing impact energy as long as the collision system behaves adiabatically.

This discussion shows that our results and interpretation very nearly coincide with the speculation which Folkmann et al (1976) made at the end of their paper. With the present calculations it becomes clear that they mainly observed the behaviour of the $8_{(1 / 2)}$ level. The main difference occurs for the $6 \cdot 3 \mathrm{MeV}$ spectrum. According to our interpretation the anisotropy peak is shifted to smaller photon energies. We interpret the experimental plateau above $7 \mathrm{keV}$ as arising from the behaviour of the minimum of the $7_{(1 / 2)}$ level at $R \cong 0.05 \mathrm{au}$, because for this impact energy the contributions from the $8_{(1 / 2)}$ level do not occur at these photon energies. The distance of closest approach is too large to reach the $8_{(1 / 2)}$ level minimum, so the lower levels dominate in the high-energy region.

\section{Conclusion}

The anisotropic emission of $\mathrm{M}$ MO $\mathrm{x}$-ray radiation in slow $\mathrm{I}-\mathrm{Au}$ collisions has been treated theoretically by the kinematic-dipole model of anisotropy for spontaneous transitions. Because of the complexity of the problem, a large number of approximations had to be introduced. The only additional information used relates to the details of the special correlation diagram involved and the relative hole distribution within the quasimolecular levels. The gross structure of the anisotropy of M MO radiation can be explained. This is additional evidence that this model is able to explain the behaviour of anisotropies of inner-shell mo radiation. 


\section{Acknowledgments}

We acknowledge helpful discussions with Professors Wölfli and Kaun as well as excellent cooperation with the Gesellschaft für Schwerionenforschung concerning experimental data and computing facilities.

\section{References}

Anholt R 1976 J. Phys. B: Atom. Molec. Phys. 9 L249 1978 Z. Phys. A 288257

Briggs J S and Dettmann K 1977 J. Phys. B: Atom. Molec. Phys. 101113

Briggs J S and Taulbjerg K 1976 J. Phys. B: Atom. Molec. Phys. 91641

Folkmann F, Armbruster P, Hagmann S, Kraft G, Mokler P H and Stein H J 1976 Z. Phys. A 27615

Frank W, Gippner P, Kaun K-H, Manfrass P and Tretyakov Yu P 1976a Z. Phys. A 277333

- 1976b Z. Phys. A 279213

Fricke B, Morović T, Sepp W-D, Rosén A and Ellis D E 1976 Phys. Lett. 59A 375

Fritsch W and Wille U 1977 J. Phys. B: Atom. Molec. Phys. 10 L165

Greenberg J S, Davis C K and Vincent P 1974 Phys. Lett. 33473

Gros M, Greenland P T and Greiner W 1977 Z. Phys. A 28031

Hagmann S 1977 Thesis Universität Köln

Hartung $\mathrm{H}$ and Fricke B 1978 Z. Phys. A 288345

Jackson J D 1975 Classical Electrodynamics 2nd edn (New York: Wiley) p 752

Kraft G, Mohler P H and Stein H J 1974 Phys. Rev. Lett. 33476

Landau L 1932 Phys. Z. SowjUn. 240

Meyerhof W E, Saylor T K and Anholt R 1975 Phys. Rev. A 122641

Morović T, Fricke B, Sepp W-D, Rosén A and Fllis D E 1978 Jap. J. Appl. Phys. to be published

Müller B and Greiner W 1974 Phys. Rev. Lett. 33469

Weisskopf V F 1932 Z. Phys. 75287

Wölfli W, Stoller Ch, Bonani G, Stöckli M and Suter M 1975 Lett. Nuovo Cim. 14577

Wölfli W, Stoller Ch, Bonani G, Suter M and Stöckli M 1977 J. Phys. B: Atom. Molec. Phys. 10 L347

Wölffi W, Stoller C, Bonani G, Suter M, Stöckli M and Däppen W 1976 Phys. Rev. Lett. 36309

Zener C 1932 Proc. R. Soc. A 137696 